\title{
Single-photon emission by semiconductor quantum rings in a photonic crystal
}

\author{
E. Gallardo, ${ }^{1, *}$ L. J. Martínez, ${ }^{2}$ A. K. Nowak, ${ }^{1}$ D. Sarkar, ${ }^{1,3}$ D. Sanvitto, ${ }^{1}$ H. P. van der Meulen, ${ }^{1}$ J. M. Calleja, ${ }^{1}$ \\ I. Prieto, ${ }^{2}$ D. Granados, ${ }^{2}$ A. G. Taboada, ${ }^{2}$ J. M. García, ${ }^{2}$ and P. A. Postigo ${ }^{2}$ \\ ${ }^{1}$ Departamento de Física de Materiales, Universidad Autónoma de Madrid, E-28049 Madrid, Spain \\ ${ }^{2}$ Instituto de Microelectrónica de Madrid, Centro Nacional de Microelectrónica, Consejo Superior de Investigaciones \\ Científicas, Isaac Newton 8, PTM Tres Cantos, E-28760 Madrid, Spain \\ ${ }^{3}$ on leave to Department of Physics and Astronomy, University of Sheffield, S3 7RH UK \\ *Corresponding author: eva.gallardo@uam.es
}

Received December 2, 2009; revised January 20, 2010; accepted January 21, 2010;

posted January 22, 2010 (Doc. ID 120804); published March 5, 2010

\begin{abstract}
Photon correlation measurements on single InAs/GaAs quantum rings embedded in a photonic crystal lattice demonstrate single-photon emission with $\mathrm{g}^{(2)}(0)$ values of 0.4 and photon antibunching between the exciton and biexciton emissions. The measured photon antibunching times of the excitons are longer than that of the biexcitons, resulting in the time asymmetry of the exciton-biexciton photon cross-correlation. Phonon sidebands due to the piezoelectric coupling of excitons to acoustic phonons broaden the emission lines and shift them to lower energies at low excitation intensity. (C) 2010 Optical Society of America OCIS codes: $030.5290,160.6000,160.5298$.
\end{abstract}

\section{INTRODUCTION}

Single-photon emission (SPE) by semiconductor quantum dots for quantum information applications has been demonstrated in past years [1-4]. The SPE signature is photon antibunching, i.e., a minimum in the second-order photon correlation function $\mathrm{g}^{(2)}(\tau)$ at zero delay time $(\tau$ $=0)$. Semiconductor quantum rings (QRs) of nanometer size are quasi-zero-dimensional structures producing total carrier confinement, as quantum dots (QDs), but showing new properties resulting from their ringlike shape [5-7]. QRs are interesting systems for physics and applications for at least two reasons. First, they display angular momentum dependent properties, such as Aharonov-Bohm type effects [8-11] including oscillatory persistent currents [12]. Second, they allow better control of their optical properties than do QDs by varying shape parameters such as ring width or outer-to-inner radius ratio. As for applications, infrared photodetectors with improved spectral range and temperature stability, compared to QD-based ones, have been reported [13]. The possible use of QRs as single-photon emitters is interesting in view of the increased flexibility in electronic structure design compared to QDs. Besides, the ability to grow QRs on photonic crystals (PCs) (and eventually on optical cavities embedded in it) opens the possibility of studying quantum electrodynamics effects using quantum rings. Photon antibunching under pulsed excitation has been reported recently [14] in GaAs/AlGaAs double QRs with $\mathrm{g}^{(2)}(0)$ values below 0.5 for ring diameters below $50 \mathrm{~nm}$.

In this paper we present second-order time-correlation measurements of the exciton $(\mathrm{X})$ and biexciton $(\mathrm{XX})$ emission lines of a single InAs/GaAs QR embedded in a PC under continuous nonresonant excitation. Unlike pulsed excitation, this method allows us to measure not only the antibunching minimum but also the characteristic time (rise time, $\tau_{\mathrm{R}}$ ) of $\mathrm{g}^{(2)}(\tau)$. Clear photon antibunching and bunching is observed by detecting coincidences in autoand cross-correlation configurations, respectively. The $\tau_{\mathrm{R}}$ value of the exciton emission is significantly larger than that of the biexciton one. This is consistent with the asymmetric shape of the $\mathrm{X}-\mathrm{XX}$ photon cross-correlation near $\tau=0$. To the best of our knowledge, this is the first experimental determination of the antibunching times in QRs.

\section{EXPERIMENT}

Self-assembled InAs QRs were grown by solid-source molecular beam epitaxy [15] inside a $158 \mathrm{~nm}$ thick GaAs slab grown on top of a $500 \mathrm{~nm}$ thick $\mathrm{Al}_{0.75} \mathrm{Ga}_{0.25} \mathrm{As}$ sacrificial layer. An atomic force microscopy (AFM) image of the uncapped layer indicates a QR surface density of $7.5 \cdot 10^{9} \mathrm{QRs} / \mathrm{cm}^{2}$ (Fig. 1). The QR height average is $2 \mathrm{~nm}$, and the average lateral size is $50 \mathrm{~nm}$ in the (1-10) direction and is $20 \%$ longer in the (110) direction, due to the anisotropy in the In diffusion coefficient. Cross sectional scanning tunneling microscope (STM) images reveal that the QR shape is maintained after capping [16]. A PC triangular lattice of holes of $140 \mathrm{~nm}$ diameter with a lattice constant of $230 \mathrm{~nm}$ was patterned by e-beam lithography and dry etching [17]. Air-suspended membranes were realized by sacrificial etching of the underlying AlGaAs layer. The PC provides a photonic bandgap for the QR emission in order to enhance the light extraction efficiency [18]. The photonic bandgap of our PC ranges from $736 \mathrm{~nm}$ to $952 \mathrm{~nm}$.

Photoluminescence (PL) spectra of single QRs were taken with a micro-PL setup perpendicular to the sample surface under nonresonant excitation using a $\mathrm{He}-\mathrm{Ne}$ laser and a CCD detector located at one of the exits of a 


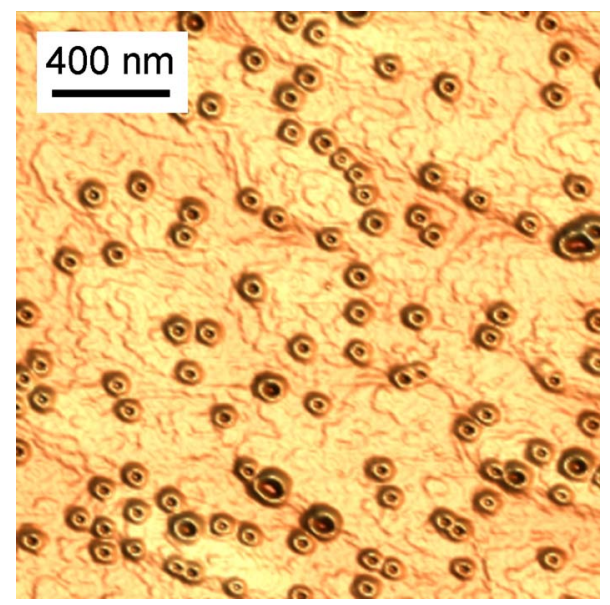

Fig. 1. (Color online) AFM image of the uncapped layer of InAs QRs.

single spectrometer. The collection aperture was NA 0.5 with a spot size $2 \mu \mathrm{m}$, and the spectrometer slits were set to a resolution of $100 \mu \mathrm{eV}$. We selected a QR in the highenergy tail of the $\mathrm{QR}$ distribution to have only a few $\mathrm{QRS}$ emitting in the laser spot. The intensity correlation measurements were done with a Hanbury-Brown and Twiss interferometer located at the second exit of the spectrometer. Two avalanche photodiodes with $30 \%$ efficiency at the QR emission wavelength $(920 \mathrm{~nm})$ were used for coincidence detection. Their response time (0.5 ns) was measured with 2 ps pulses of a Ti-sapphire laser. Count rates at the detectors were around 3000 counts/s - a factor of 6 stronger than QRs located outside the PC. All measurements were performed at $7 \mathrm{~K}$.

\section{RESULTS AND DISCUSSION}

PL spectra of a single $\mathrm{QR}$ for different excitation powers are shown in Fig. 2. Three emission peaks are identified according to their intensity $I$ versus excitation power $P$ dependence $\left(I \propto P^{\gamma}\right)$. We obtain $\gamma=1.02$ for $\mathrm{X}, \gamma=1.87$ for $\mathrm{XX}$, and $\gamma=1.39$ for the peak at $1.355 \mathrm{eV}$. According to its power dependence and its energy relative to $\mathrm{X}$ and $\mathrm{XX}$, we attribute the peak at $1.355 \mathrm{eV}$ to the positive trion $\mathrm{X}^{+}$.

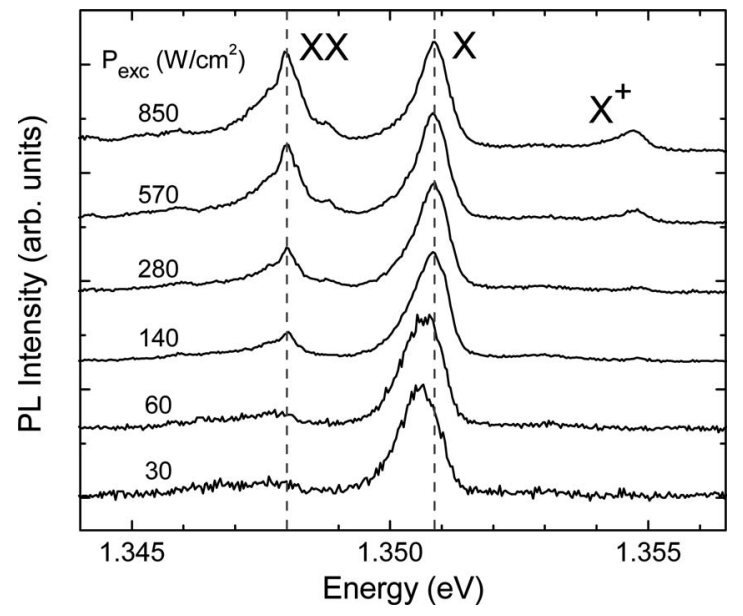

Fig. 2. Power-dependent PL spectra (normalized to the $\mathrm{X}$ line intensity) of a single InAs/GaAs QR.
The peak widths of $\mathrm{X}$ and $\mathrm{XX}$ are rather large $(0.7 \mathrm{meV})$ compared to QD excitons, similarly as reported for GaAs QRs [15]. The origin of this large width is not known, but it is possibly related to spectral diffusion. The $\mathrm{X}$ and $\mathrm{XX}$ line shapes change significantly upon decreasing excitation power: they broaden and slightly red-shift. This behavior has been reported for InAs/AlAs QDs and explained in terms of sidebands due to acoustic phonon emission [19]. Increasing the excitation power screens out the piezoelectric coupling of $\mathrm{X}$ and $\mathrm{XX}$ to acoustic phonons, leading to a transition from broad spectra dominated by low-energy phonon sidebands to narrower lines corresponding to the zero-phonon-line exciton emission. The $\mathrm{X}$ and $\mathrm{XX}$ emission energies do not show any observable dependence on linear polarization. We can estimate an upper limit for the exciton fine structure splitting [20] of $70 \mu \mathrm{eV}$.

The second-order photon autocorrelation function of the exciton is presented in Fig. 3(a). The solid curve is a fit to the standard expression

$$
g^{(2)}(\tau)=1-\beta \exp \left(-|\tau| / \tau_{R}\right)
$$

convoluted with the time-response function of the detectors. The function found for our detectors was of the form $\exp (-|\tau| / \sigma)$, with a response time $\sigma$ of $0.5 \mathrm{~ns}$. The deconvoluted values found are $\mathrm{g}^{(2)}(0)=0.42$ and $\tau_{\mathrm{R}}=1.2 \mathrm{~ns}$, corresponding to the dashed curve in Fig. 3(a). As the signalto-noise ratio is typically above 0.9 , the background [21] contribution to $\mathrm{g}^{2}(0)$ is small. Subtraction of this contribution gives $\mathrm{g}^{(2)}(0)=0.34$ and the same $\tau_{\mathrm{R}}$. A similar antibunching behavior has been observed in few QRs with antibunching times ranging from 1 to $2.5 \mathrm{~ns}$. The corresponding values for the $\mathrm{XX}$ autocorrelation function (not shown) are $\mathrm{g}^{(2)}(0)=0.27$ and $\tau_{\mathrm{R}}=0.6 \mathrm{~ns}$. The $\mathrm{g}^{(2)}(0)$

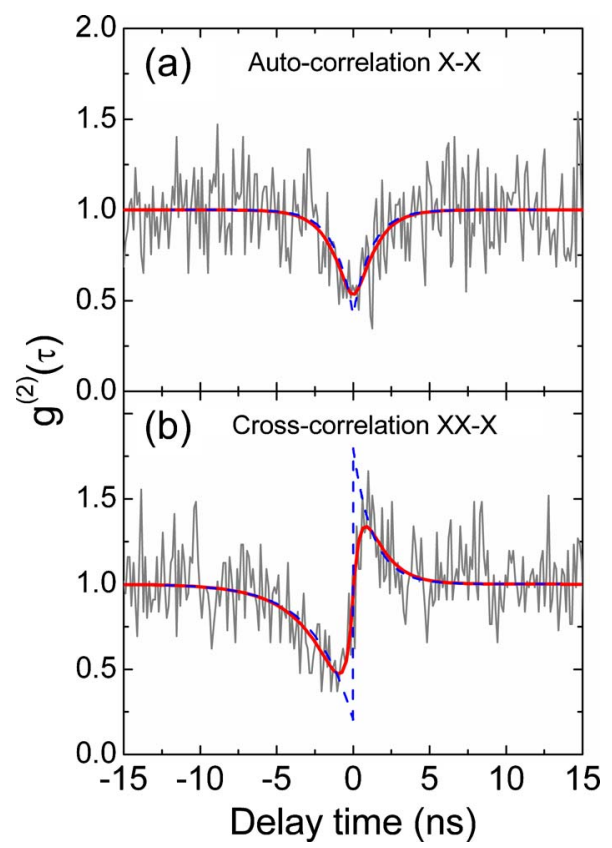

Fig. 3. (Color online) (a) Autocorrelation function of the $\mathrm{X}$ emission and (b) cross-correlation function between X and XX emissions of a single InAs/GaAs $\mathrm{QR}$. The red continuous curves represent the fits convoluted with the detector response function; the blue dashed curves represent the unconvoluted fits. 
values below 0.5 indicate $\mathrm{SPE}$. The XX-X crosscorrelation function is shown in Fig. 3(b). Start and stop signals are given by the $\mathrm{XX}$ and $\mathrm{X}$ photon detection, respectively. The asymmetric shape is characteristic of the biexciton-exciton emission cascade, as the exciton emission probability is increased (decreased) right after (before) biexciton emission. The full line-shape analysis requires solving a rate equation system involving several QR states with up to nine parameters [22,23]. Instead, we have used a simple exponential fit (solid curve) to have an estimate of the involved times. The left-hand side of Fig. 3 (b) shows a characteristic time of $2.4 \mathrm{~ns}$, whereas the right-hand side shows a faster evolution $\left(\tau_{R}=1.3 \mathrm{~ns}\right)$. This time asymmetry is the consequence of the different rise times found in the autocorrelation functions of $\mathrm{X}$ and $\mathrm{XX}$. For an empty $Q R$, the capture of an electron-hole pair can result in either a bright (angular momentum \pm 1 ) or a dark (angular momentum \pm 2 ) exciton. Instead, the capture of a second $\mathrm{e}-\mathrm{h}$ pair by any exciton (either bright or dark) always results in an optically active biexciton. As the excitation intensity in our experiments is set to have almost equal X and XX PL intensities, the probability of emitting two consecutive $\mathrm{X}$ photons with short time delay is expected to be lower than between XX photons. The same qualitative argument applies to the different $\tau_{\mathrm{R}}$ values for $\tau<0$ and $\tau>0$ observed in Fig. 3(b). The emission probability of a XX photon shortly after an $\mathrm{X}$ one should be smaller than the other way around. After emission of a $\mathrm{XX}$ photon, the $\mathrm{QR}$ is immediately ready to emit an $\mathrm{X}$ one, but after $\mathrm{X}$ emission the $\mathrm{QR}$ has to be charged again with two electron-hole pairs before emitting a XX photon.

\section{CONCLUSIONS}

In summary, we present evidence of single-photon emission by InAs quantum rings inside a photonic crystal lattice, given by auto- and cross-correlation measurements of the exciton and biexciton transitions. The line shapes of the different $\mathrm{g}^{(2)}(\tau)$ functions indicate characteristic times longer for the exciton emission than for the biexciton one for similar emission intensities. At low excitation powers, the spectral shapes of both $\mathrm{X}$ and $\mathrm{XX}$ emission lines are dominated by acoustic phonon sidebands due to their piezoelectric coupling to excitons.

\section{ACKNOWLEDGMENTS}

The authors are indebted to $\mathrm{E}$. $\mathrm{Hu}$ and $\mathrm{R}$. Sabouni for helpful discussions. This work has been supported by research contracts of the Spanish Ministry of Education grants MAT2008-01555/NAN and Consolider CSD 200619, and the Community of Madrid grants CAM S-0505ESP-0200 and Naninpho-QD TEC2008-06756-C03-01.

\section{REFERENCES}

1. P. Michler, A. Imamoglu, M. D. Mason, P. J. Carson, G. F. Strouse, and S. K. Buratto, "Quantum correlation among photons from a single quantum dot at roomtemperature," Nature 406, 968-970 (2000).
2. B. Lounis, H. A. Bechtel, D. Gerion, P. Alivisatos, and W. E. Moerner, "Photon antibunching in single CdSe/ZnS quantum dot fluorescence," Chem. Phys. Lett. 329, 399-404 (2000).

3. C. Santori, M. Pelton, G. Solomon, Y. Dale, and Y. Yamamoto, "Triggered single photons from a quantum dot," Phys. Rev. Lett. 86, 1502-1505 (2001)

4. V. Zwiller, H. Blom, P. Jonsson, N. Panev, S. Jeppesen, T. Tsegaye, E. Goobar, M.-E. Pistol, L. Samuelson, and G. Björk, "Single quantum dots emit single photons at a time: antibunching experiments," Appl. Phys. Lett. 78, 24762478 (2001)

5. R. J. Warburton, C. Schäflein, D. Haft, F. Bickel, A. Lorke, K. Karrai, J. M. Garcia, W. Schoenfeld, and P. M. Petroff, "Optical emission from a charge-tunable quantum ring," Nature 405, 926-929 (2000).

6. D. Granados, J. M. García, T. Ben, and S. I. Molina, "Vertical order in stacked layers of self-assembled $\operatorname{In}(\mathrm{Ga})$ As quantum rings on GaAs (001)," Appl. Phys. Lett. 86, 071918 (2005).

7. B. Alén, J. Martínez-Pastor, D. Granados, and J. M. García, "Continuum and discrete excitation spectrum of single quantum rings," Phys. Rev. B 72, 155331-155336 (2005).

8. A. Lorke, R. Luyken, A. O. Govorov, J. P. Kotthaus, J. M. Garcia, and P. M. Petroff, "Spectroscopy of nanoscopic semiconductor rings," Phys. Rev. Lett. 84, 2223-2226 (2000).

9. M. Bayer, M. Korkusinski, P. Hawrylak, T. Gutbrod, M. Michel, and A. Forchel,"Optical detection of the AharonovBohm effect on a charged particle in a nanoscale quantum ring," Phys. Rev. Lett. 90, 186801-186804 (2003).

10. A. O. Govorov, S. E. Ulloa, K. Karrai, and R. J. Warburton, "Polarized excitons in nanorings and the optical AharonovBohm effect," Phys. Rev. B 66, 0813091-0813094(R) (2002).

11. V. M. Fomin, V. N. Gladilin, S. N. Klimin, J. T. Devreese, N. A. J. M. Kleemans, and P. M. Koenraad, "Theory of electron energy spectrum and Aharonov-Bohm effect in selfassembled $\mathrm{In}_{\mathrm{x}} \mathrm{Ga}_{1-x} \mathrm{As}$ quantum rings in GaAs," Phys. Rev. B 76, 235320 (2007)

12. N. A. J. M. Kleemans, I. M. A. Bominaar-Silkens, V. M. Fomin, V. N. Gladilin, D. Granados, A. G. Taboada, J. M. García, P. Offermans, U. Zeitler, P. C. M. Christianen, J. C. Maan, J. T. Devreese, and P. M. Koenraad, "Oscillatory persistent currents in self-assembled quantum rings," Phys. Rev. Lett. 99, 146808 (2007).

13. H. S. Ling, S. Y. Wang, C. P. Lee, and M. C. Lo, "Characteristics of $\operatorname{In}(\mathrm{Ga})$ As quantum ring infrared photodetectors," J. Appl. Phys. 105, 034504 (2009).

14. M. Abbarchi, C. A. Mastrandrea, A. Vinattieri, S. Sanguinetti, T. Mano, T. Kuroda, N. Koguchi, K. Sakoda, and M. Gurioli, "Photon antibunching in double quantum ring structures," Phys. Rev. B 79, 085308 (2009).

15. D. Granados and J. M. García, "In(Ga)As self-assembled quantum ring formation by molecular beam epitaxy," Appl. Phys. Lett. 82, 2401-2403 (2003).

16. P. Offermans, P. M. Koenraad, J. H. Wolter, D. Granados, J. M. García, V. M. Fomin, V. N. Gladilin, and J. T. Devreese, "Atomic-scale structure of self-assembled $\operatorname{In}(\mathrm{Ga})$ As quantum rings in GaAs," Appl. Phys. Lett. 87, 131902 (2005).

17. K. Hennessy, A. Badolato, P. M. Petroff, and E. Hu, "Positioning photonic crystal cavities to single InAs quantum dots," Photonics Nanostruct. Fundam. Appl. 2, 65-72 (2004).

18. M. Kaniber, A. Laucht, T. Hürlimann, M. Bichler, R. Meyer, M.-C. Amann, and J. J. Finley, "Highly efficient singlephoton emission from single quantum dots within a twodimensional photonic band-gap," Phys. Rev. B 77, 073312 (2008).

19. D. Sarkar, H. P. van der Meulen, J. M. Calleja, J. M. Meyer, R. J. Haug, and K. Pierz,"Piezoelectric exciton acousticphonon coupling in single quantum dots," Phys. Rev. B 78, 241305 (2008)

20. D. Gammon, E. S. Snow, B. V. Shanabrook, D. S. Katzer, and D. Park, "Fine structure splitting in the optical spectra of single GaAs quantum dots," Phys. Rev. Lett. 76, 30053008 (1996). 
21. R. Brouri, A. Beveratos, J. P. Poizat, and P. Grangier, "Photon antibunching in the fluorescence of individual color centers in diamond," Opt. Lett. 25, 1294-1296 (2000).

22. C. Santori, D. Fattal, J. Vucovic, G. S. Solomon, E. Waks, and Y. Yamamoto,"Submicrosecond correlations in photolu- minescence from InAs quantum dots," Phys. Rev. B 69, 205324 (2004).

23. M. H. Baier, A. Malko, E. Pelucchi, D. Y. Oberli, and E. Kapon,"Quantum-dot exciton dynamics probed by photoncorrelation spectroscopy,” Phys. Rev. B 73, 205321 (2006). 